Case Report

\title{
Veno-Veno Extracorporeal Membrane Oxygenation in Post-Traumatic Acute Lung Injury
}

\author{
Se Heon Kim a, Young Hoon Sul a,b,*, Jin Young Lee a, Jin Bong Ye a, Jin Suk Lee a, Soo Young Yoon ${ }^{c}$, Joong Suck Kim ${ }^{\text {d }}$ \\ a Department of Trauma Surgery, Chungbuk National University Hospital, Cheongju, Korea \\ ${ }^{b}$ Department of Trauma Surgery, College of Medicine, Chungbuk National University, Cheongju, Korea \\ ${ }^{c}$ Department of Thoracic and Cardiovascular Surgery, Chungbuk National University Hospital, Cheongju, Korea, \\ ${ }^{d}$ Department of Trauma Surgery, Cheju Halla General Hospital, Jeju, Korea
}

\section{Article history:}

Received: August 16, 2019

Revised: October 24, 2019

Accepted: November 11, 2019

*Corresponding Author:

Young Hoon Sul

Department of Trauma Surgery, College of

Medicine, Chungbuk National University,

Cheongju, Korea

E-mail: ssulyh@gmail.com

\section{ORCID}

Se Heon Kim

https://orcid.org/0000-0002-1864-4096

Young Hoon Sul

https://orcid.org/0000-0003-3184-3396

Jin Young Lee

https://orcid.org/0000-0002-4629-0934

Jin Bong Ye

https://orcid.org/0000-0002-5597-4502

Jin Suk Lee

https://orcid.org/0000-0002-6694-4683

Soo Young Yoon

https://orcid.org/0000-0001-9264-1371

Joong Suck Kim

https://orcid.org/0000-0003-4498-354X

\begin{abstract}
Patients with severe lung injury and hemorrhagic shock, may develop acute respiratory failure syndrome during resuscitation, and may require extracorporeal membrane oxygenation (ECMO) support to ensure adequate oxygenation to sustain life. In this case study, a 69 year-old female was hit by a motor vehicle whilst riding her bicycle. She was in a state of hemorrhagic shock due to polytrauma and was resuscitated with massive fluid transfusion during the initial management. After admission to the intensive care unit, she suffered from hypoxia which required mechanical ventilation using $100 \%$ oxygen. However, hypoxia did not improve, so she was placed on venoveno ECMO support after 4 days of intensive care treatment. Although trauma and bleeding are considered as relative contraindications for ECMO support, veno-veno ECMO allows for lung rest, and improvement of pulmonary function.
\end{abstract}

Keywords: acute lung injury, extracorporeal membrane oxygenation, mechanical ventilator, trauma

\section{Introduction}

Lung contusion caused by blunt chest trauma is usually treated conservatively. However, patients with severe lung injury and hemorrhagic shock may develop acute respiratory failure syndrome during resuscitation [1]. Mechanical ventilation used for conventional management of gas exchange has its limits and patients may require the initiation of extracorporeal membrane oxygenation (ECMO) for adequate oxygenation [2]. A successful outcome of ECMO treatment for a patient with severe lung injury and hemorrhagic shock is presented in this case study.

\section{Case Report}

This study was approved by the Institutional Review Board of Chungbuk National University Hospital (approval no.: 2019-09010).

A 69-year-old female was brought to Chungbuk regional trauma center after being hit by a motor vehicle whilst riding her bicycle. She was initially in a coma when her vital signs were recorded (blood pressure $45 / 30 \mathrm{mmHg}$, heart rate 130 beats/minute, respiration rate 45 times/minute, and body temperature $35.7^{\circ} \mathrm{C}$ ). Immediately after tracheal intubation, fluid resuscitation was performed using 3 units of emergency 


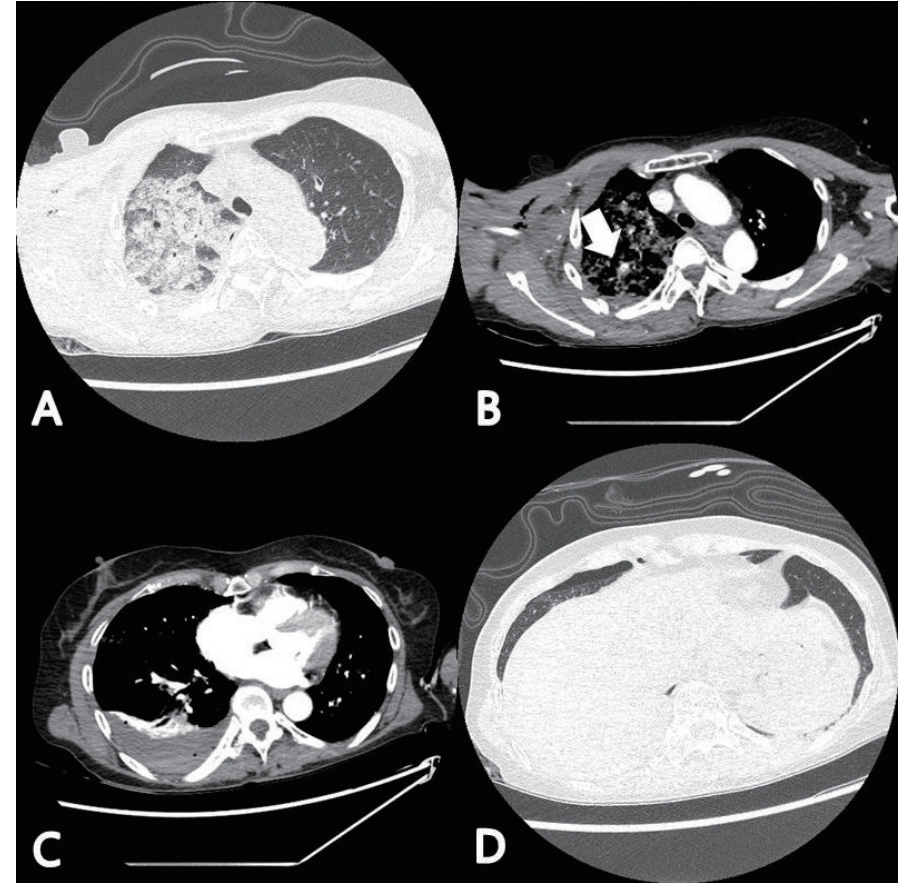

Figure 1. Initial computed tomographic scan findings. (A) Severe lung contusion in right lung field, (B) petechial hemorrhage with extravasation in right lung field, (C) hemothorax of right lung, (D) minimal pneumothorax of right lung.

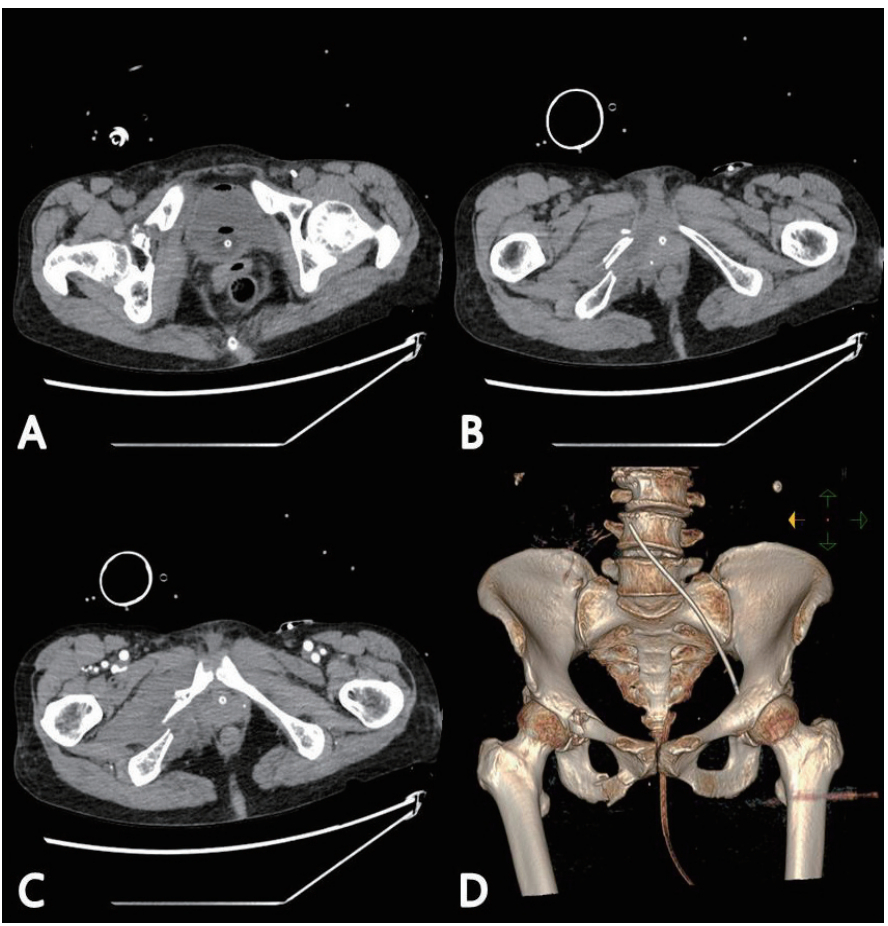

Figure 3. Unstable fracture of the pelvic bone. (A) Superior ramus fracture (B) and (C) inferior ramus fracture (D) 3-dimensional reconstruction of pelvic fracture.

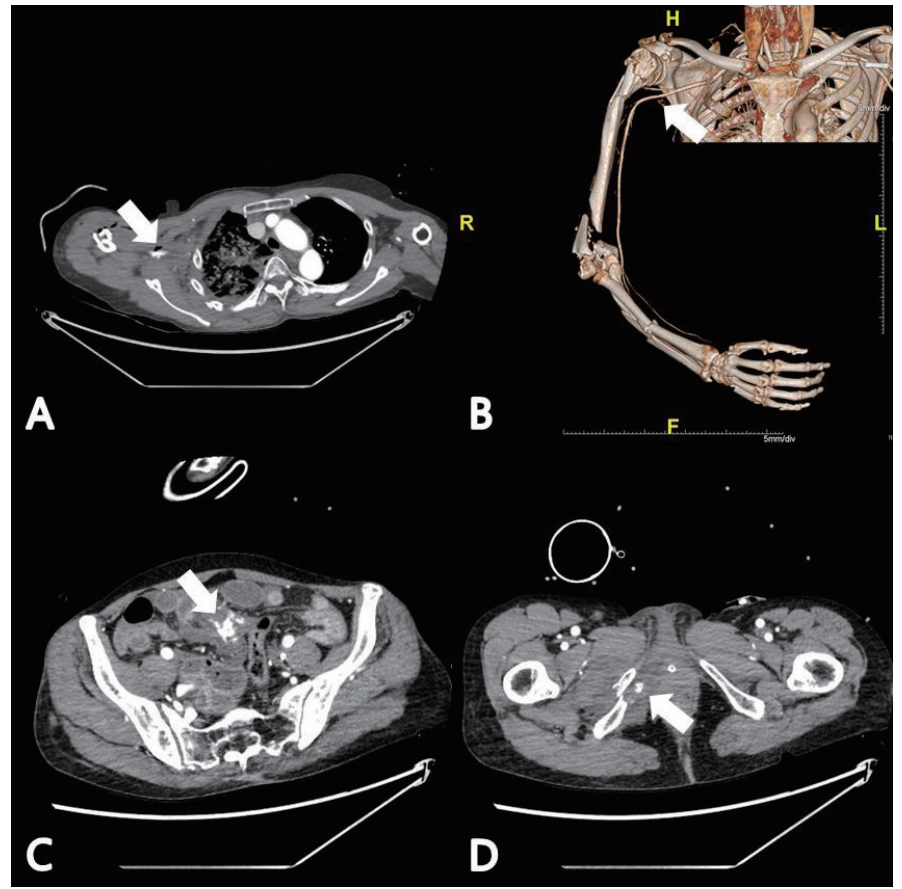

Figure 2. (A) Focal traumatic pseudoaneurysm with bulging in the right axillary artery, $(B)$ reconstructed 3 -dimensional image of multiple fractures in the right humerus with degloving injury on the right arm, (C) contrast media leakage in the small bowel mesentery with hemoperitoneum, (D) contrast media leakage at the fracture of the right superior and inferior pubic rami.

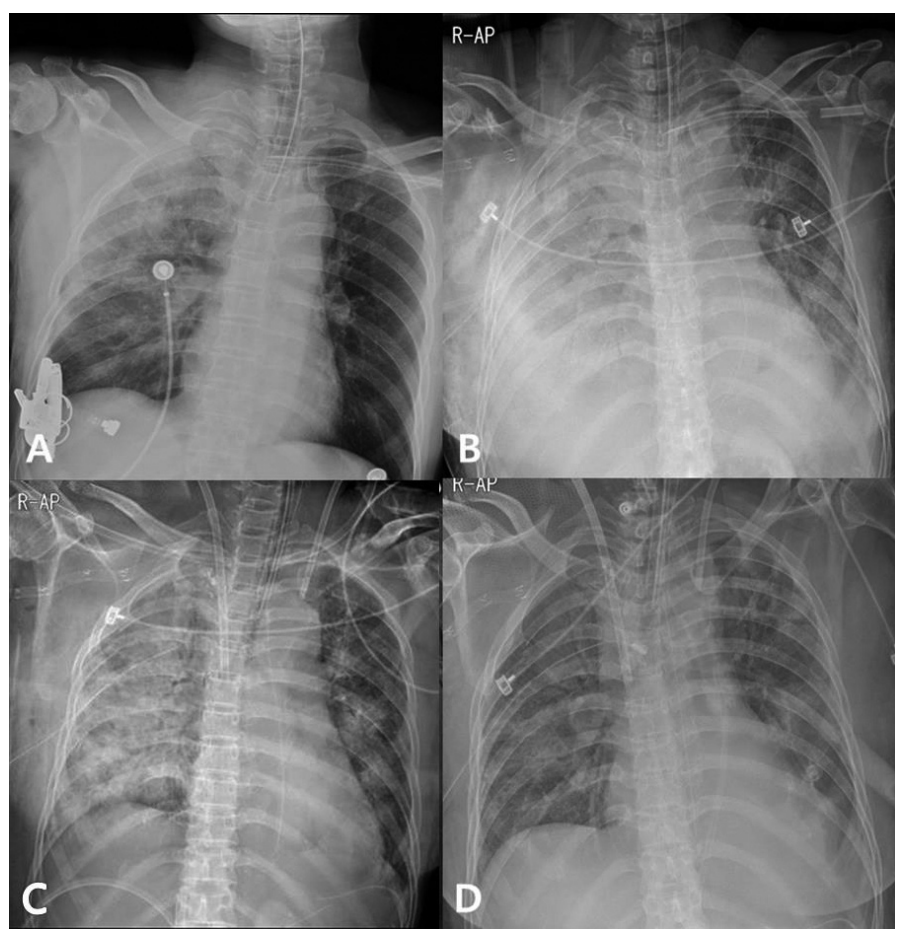

Figure 4. Chest X-rays showing clinical progression. (A) Initial X-ray, (B) severe pulmonary edema and pleural effusion are worsened after 12 hours of hospitalization, (C) veno-veno extracorporeal membrane oxygenation was applied, (D) pulmonary edema was improved after veno-veno extracorporeal membrane oxygenation 4 days after hospitalization. 
transfusion, and radiological evaluations were carried out. Computed tomography scans demonstrated minimal traumatic subarachnoidal hemorrhage, laceration with pseudoaneurysm of the brachial artery, open humerus fracture, multiple rib fractures with pneumohemothorax, active bleeding from lung parenchyma along with lung contusion, mesenteric injury with active arterial bleeding, and unstable pelvic fracture (Figures 1-3). Immediately following radiological evaluation a chest tube was inserted to treat the hemothorax, and 660 $\mathrm{mL}$ of fresh blood was drained. In the initial blood test, the hemoglobin level was measured as $8.2 \mathrm{~g} / \mathrm{dL}$. To manage the patient's blood pressure, a massive transfusion protocol with vasopressor was performed. Multiple, concurrent active bleeding sites were located (brachial artery, lung parenchyma, mesentery, and pelvis) and treated using angio-embolization. Just prior to taking an angiography, the patient's heart stopped (due to hemorrhagic shock), so chest compressions were performed, and a massive transfusion protocol was initiated whilst embolizing the arteries. Following embolization of the mesentery with glue, and the pelvis with glue and coiling, circulation recovered. Systolic blood pressure was $50 \mathrm{mmHg}$ and the heart rate was 147 beats/minute. Subsequently, embolization of the lung parenchymal artery branch and intercostal artery was performed using glue. Stent insertion for the brachial arterial pseudoaneurysm was then performed. However, the brachial artery was continuously ruptured and it took 4 attempts to insert the stent successfully. After embolization was complete, her blood pressure was $83 / 40$ $\mathrm{mmHg}$, heart rate was 136 beats/minutes, respiration rate 24 breaths/minute, and oxygen saturation was at $9 \%$. She was taken to the trauma intensive care unit for treatment with mechanical ventilation and correction of disseminated intravascular coagulation. A chest X-ray 12 hours after admission, showed serious lung injury (Figure 4). Her oxygen saturation was $77 \%$ and $\mathrm{PaO} 2 / \mathrm{FiO} 2$ was below 50 , even with mechanical ventilation (pressure control mode) at $100 \%$ oxygen, and full sedation with muscle relaxant. Alveolar recruitment maneuver was ineffective, and mechanical ventilation did not provide adequate gas exchange to alleviate hypoxia, so ECMO was initiated. Following veno-veno ECMO support, her $\mathrm{PaO} 2 / \mathrm{FiO} 2$ (0.6) was 96, and oxygen saturation was $100 \%$. After 3 hours of veno-veno ECMO, abdominal bloating deteriorated and her hemoglobin level decreased from 10 to 7.6, and so an exploratory laparotomy was performed. A hematoma and a large amount of ascites (presumably due to the initial injury) were observed, but there was no overt ongoing bleeding or organ damage observed. Surgery to remove the hematoma and ascites was successful. Four days after admission, external fixation for an unstable pelvic fracture, and amputation of an open humerus fracture were performed, after which the patient was stabilized. Five days after ECMO support, her PaO2/FiO2 (0.4) was 92, and oxygen saturation was $100 \%$. A chest X-ray showed improvement in lung injury, so ECMO support was discontinued (Figure 4). During mechanical ventilation treatment, the patient developed ventilator-associated pneumonia, and was therefore weaned off ventilation (24 days after admission). At Day 42 after admission, she was transferred to the general ward where she received rehabilitation and was discharged from hospital.

\section{Discussion}

Polytrauma patients suffer from a broad range of multiple injuries. In traumatic lung injuries, severe complications such as hemorrhagic shock and acute respiratory failure may occur [1]. Lung injury is not the only cause of acute respiratory failure; performing a massive transfusion protocol for hemorrhagic shock may also cause acute respiratory failure [3].

In treating acute respiration failure, mechanical ventilation may not be sufficient, and thus ECMO support may be considered [2]. Donald Hill reported ECMO treatment for respiratory failure after trauma in 1972 [4], with the development of the ECMO technique progressing from that initial report. Although the use of ECMO support may worsen coagulopathy in patients with hemorrhagic shock $[5,6]$, ECMO has been reported for its efficacy in patients with polytrauma, including lung contusion and bronchial injuries [2,7].

The patient in this case study required immediate treatment for active bleeding, with unstable vital signs and hemorrhagic shock due to multiple injuries. Active bleeding of the abdomen and pelvis would have indicated immediate surgical treatment, but in this case bleeding of the brachial artery, and pulmonary parenchyma were considered very serious, and concurrent treatment via angiography was required. After moving the patient to the angiography room, the patient had a cardiac arrest. At that time, the medical staff judged the cause of the cardiac arrest to be hemorrhagic shock caused by multiple concurrent bleeding. Embolization was performed during massive transfusion, fluid resuscitation, and cardiopulmonary resuscitation. After embolizing the mesentery and pelvis, the return of spontaneous circulation was possible.

ECMO support has a high risk of causing bleeding, and thrombocytopenia can occur with the use of heparin, both of which are relative contraindications in patients with active bleeding.[8] In this case, active bleeding was controlled when respiratory failure worsened, and following embolization, the vital signs remained stable, with a relatively low risk of additional bleeding even with ECMO support. Even if bleeding occurred, it was considered an inevitable risk.

In the case of acute respiratory failure, as in this current case, several treatments may be used in an attempt to reduce 
hypoxia. Sedative-analgesic medications add a neuromuscular blockade and can be used to help reduce mechanical ventilation and oxygen consumption [9]. Prone position or the use of nitric oxide gas may also help reduce hypoxia [10,11]. In this case, the patient was treated with sedative-analgesic medications during mechanical ventilation, and alveolar recruitment was attempted using neuromuscular blockade to reduce hypoxia, but it was not effective. ECMO support was the only way to improve oxygenation of the patient in the absence of a nitric oxide delivery system. If the patient was placed in a prone position with abdominal distension due to intraperitoneal bleeding to attempt a reduction in hypoxia, the abdominal pressure would be increased and the diaphragm would be elevated, which would not improve the patient's condition. In addition, multiple rib fractures and unstable pelvic fractures are a constraint to abandon the prone position. Moreover, a massive transfusion (20 units of red blood cells, 10 units of fresh frozen plasma, and 10 units of platelet concentrates) was infused, which probably weakened respiration, thus it was expected that ECMO support would greatly aid in the normalization of respiration by providing sufficient time for lungs to rest and recover.

In this case, bleeding due to mesenteric injury was encapsulated in the initial computed tomography scan, and after embolization the patient's condition remained stable and so abdominal surgery was not performed immediately because it was judged that the bleeding was not progressing. In addition, mesentery injury and embolization caused by trauma could not rule out the development of bowel ischemia, and disseminated intravascular coagulation correction was performed first, and then surgery would be performed if bowel ischemia symptoms presented, peritonitis, or if delayed bleeding occurred.

Abdominal distension increased 3 hours after initiating ECMO, and this warranted an exploratory laparotomy. There was no active bleeding, so the hematoma was removed, and the abdomen was closed. The abdominal distension in the patient was not considered to be caused by an increase in bleeding due to ECMO support, and was thought to be due to a hematoma caused by the initial injury and buildup of ascites during treatment. Ventilation allowed improvement of the patient's a pulmonary edema and contusion regression, leading to ECMO support being discontinued 5 days after initiation of the treatment. Since long-term antibiotic treatment has been combined to manage ventilator associated pneumonia, successful mechanical ventilator weaning was possible 24 days after admission.

In conclusion, when acute respiratory failure syndrome occurs due to severe lung injury with hemorrhagic shock, lung rest and improvement of pulmonary function can be expected by using the veno-veno ECMO support technique to provide an adequate amount of gas exchange to sustain life.

\section{Conflicts of Interest}

No potential conflict of interest relevant to this article was reported.

\section{References}

[1] Arlt M, Philipp A, Voelkel S, Rupprecht L, Mueller T, Hilker M, et al. Extracorporeal membrane oxygenation in severe trauma patients with bleeding shock. Resuscitation 2010;81(7):804-9.

[2] Madershahian N, Wittwer T, Strauch J, Franke UF, Wippermann J, Kaluza M, et al. Application of ECMO in multitrauma patients with ARDS as rescue therapy. J Card Surg 2007;22(3):180-4.

[3] Toy P, Popovsky MA, Abraham E, Ambruso DR, Holness LG, Kopko PM, et al. Transfusion-related acute lung injury: Definition and review. Crit Care Med 2005;33(4):721-6.

[4] Hill JD, O'Brien TG, Murray JJ, Dontigny L, Bramson ML, Osborn JJ, et al. Prolonged Extracorporeal Oxygenation for Acute Post-Traumatic Respiratory Failure (Shock-Lung Syndrome) - Use of the Bramson Membrane Lung. N Engl J Med 1972;286(12):629-34.

[5] Wydo S, George R. Extracorporeal membrane oxygenation: A trauma surgeon's perspective. Mech Circ Support 2013;4(1):11826582.

[6] Wu MY, Lin PJ, Tseng YH, Kao KC, Hsiao HL, Huang CC. Venovenous extracorporeal life support for posttraumatic respiratory distress syndrome in adults: The risk of major hemorrhages. Scand J Trauma Resusc Emerg Med 2014;22:56.

[7] Campione A, Agostini M, Portolan M, Alloisio A, Fino C, Vassallo G. Extracorporeal membrane oxygenation in respiratory failure for pulmonary contusion and bronchial disruption after trauma. J Thorac Cardiovasc Surg 2007;133(6):1673-4.

[8] Sklar MC, Sy E, Lequier L, Fan E, Kanji HD. Bleeding, Transfusion, and Mortality on Extracorporeal Life Support: ECLS Working Group on Thrombosis and Hemostasis. Ann Am Thorac Soc 2016;13(12):2242-50.

[9] deBacker J, Hart N, Fan E. Neuromuscular Blockade in the 21st Century Management of the Critically Ill Patient. Chest 2017;151(3):697-706.

[10] Guérin C, Reignier J, Richard JC, Beuret P, Gacouin A, Boulain T, et al. Prone positioning in severe acute respiratory distress syndrome. N Engl J Med 2013;368(23):2159-68.

[11] Adhikari NK, Burns KE, Friedrich JO, Granton JT, Cook DJ, Meade MO. Effect of nitric oxide on oxygenation and mortality in acute lung injury: Systematic review and meta-analysis. BMJ 2007;334(7597):779. 